\title{
Chemical characterization by GC-MS and in vitro activity against Candida albicans of volatile fractions prepared from Artemisia dracunculus, Artemisia abrotanum, Artemisia absinthium and Artemisia vulgaris
}

\author{
Diana Obistioiu ${ }^{1}$, Romeo T Cristina ${ }^{1 *}$, Ivo Schmerold², Remigius Chizzola ${ }^{3}$, Klaus Stolze ${ }^{2}$, lleana Nichita ${ }^{1}$ \\ and Viorica Chiurciu ${ }^{4}$
}

\begin{abstract}
Background: A large number of essential oils is reported to have significant activity against Candida albicans. But the different chemical composition influences the degree of their activity. The intention of this study was to investigate the chemical composition and the activity against Candida albicans of volatile oils obtained from Artemisia dracunculus, A. abrotanum, A. absinthium and A. vulgaris (Asteraceae). The aim of the study was to identify new chemical compounds that have effect against $C$. albicans.

The essential oils were obtained by hydrodistillation or extraction with dichloromethane (a new procedure we developed trying to obtain better, more separated compounds) from air dried above ground plant material and analyzed by GC-MS. Additionally commercial essential oils from the same species were tested. The Candida albicans inhibition studies were carried out by the paper disc diffusion method.

Results: The essential oils shared common components but presented differences in composition and showed variable antifungal activity. Davanone and derivatives thereof, compounds with silphiperfolane skeleton, estragole, davanone oil, $\beta$-thujone, sabinyl acetate, herniarin, cis-chrysanthenyl acetate, 1,8-cineol, and terpineol were the main components of Artemisia volatiles.

Conclusions: Among the volatile fractions tested those from A. abrotanum containing davanone or silphiperfolane derivatives showed the highest antifungal activity. The in vitro tests revealed that the Artemisia oils are promising candidates for further research to develop novel anti-candida drugs.
\end{abstract}

Keywords: Artemisia, Essential oils, GC-MS, Antifungal activity, Candida albicans

\section{Background}

The genus Artemisia belongs to one of the largest and most widely distributed genera of the family Asteraceae (Compositae). It is a diverse and economically important genus and it has more than 500 species. Most plants within this genus have a great importance as medication,

\footnotetext{
*Correspondence: rtcristina@yahoo.com

'Banat's University of Agricultural Sciences and Veterinary Medicine from Timisoara, Faculty of Veterinary Medicine, Calea Aradului no 119, Timisoara 300645, Romania

Full list of author information is available at the end of the article
}

foodstuff, ornamentals or soil stabilizers, some are allergenic or toxic, and some are weeds growing in the fields [1-4].

Antimycotic activity of $A$. absinthium against Candida albicans was reported in several in vitro studies [5,6] however, there are also investigations that found no such activity $[7-10]$.

The conditions that may separate these studies are the plant preparations put under investigation. The highest percentage of successful antifungal activity of Artemisia

\section{() Chemistry Central}

(c) 2014 Obistioiu et al.; licensee Chemistry Central Ltd. This is an Open Access article distributed under the terms of the Creative Commons Attribution License (http://creativecommons.org/licenses/by/2.0), which permits unrestricted use, distribution, and reproduction in any medium, provided the original work is properly credited. The Creative Commons Public Domain Dedication waiver (http://creativecommons.org/publicdomain/zero/1.0/) applies to the data made available in this article, unless otherwise stated. 
plants was obtained with essential oils; raw extracts generally showed less activity [5-10].

More information on the potential antifungal activity of Artemisia plants could stimulate research leading to their possible future use in the clinical treatment of candidiasis as natural antimycotic agents.

\section{Results and discussion}

In the present study, the chemical composition and the antifungal in vitro potency of seven oil preparations obtained from air dried plants and commercial essential oil samples of 4 different Artemisia species were put under investigation. Their chemical composition was evaluated by GC-MS. The antifungal activity was determined by the disk diffusion method test. The tests were done using the disc diffusion method following CLSI standard rules for antimicrobial susceptibility testing of impregnated disks [11].

\section{Chemical composition}

The volatile fractions studied showed a great chemical diversity. All four Artemisia species had distinct volatile patterns and there were also differences in the composition between the two different extracts, the distilled oil and the $\mathrm{CH}_{2} \mathrm{Cl}_{2}$ extract, of the same species, as presented in Table 1.

A. abrotanum was characterized by the high content of the sesquiterpene davanone and its derivatives which could not all be identified as well as by the artedouglasia oxides A-D. Monoterpenes like 1-terpineol or trans-piperitol and the phenylpropanoid estragole were present in small amounts only in this species. All main compounds found in the distilled oil were also present in the $\mathrm{CH}_{2} \mathrm{Cl}_{2}$ extract. The davanone derivatives were also the main compounds in the microdiostillates which also contained the artedouglasia oxides.

The main compound in the distilled oil of $A$. dracunculus was estragole accounting for approximately $80 \%$ of the oil. However, in the $\mathrm{CH}_{2} \mathrm{Cl}_{2}$ extract this compound could not be identified. Small amounts of the phenylpropanoids chavicol and methyleugenol could be found. These extracts contained also some davanone and its derivatives. Both, the distilled oil and the extract contained the coumarin derivative herniarin. In the microdistillate, the main compounds were estragole $(79.5 \%)$ followed by $Z-\beta$-ocimene $(8.8 \%)$ and $E$ - $\beta$-ocimene (7.2\%).

From $A$. absinthium only a $\mathrm{CH}_{2} \mathrm{Cl}_{2}$ extract was obtained. The main peak in the chromatogram could not be identified. Furthermore, the extract contained $\beta$-thujone and nerol as major compounds and estragole, spathulenol and $\alpha$-bisabolol as further components. Herniarin was also present in this extract. The microdistillate was characterized by the presence of $\beta$-thujone (78.1\%), sabinene (4.4\%), and myrcene (4.4\%).
The main volatiles in A. vulgaris were terpinen-4-ol, and borneol. One noticeable difference between the two volatile preparations was that 1,8 -cineol, the main compound in the oil from the $\mathrm{CH}_{2} \mathrm{Cl}_{2}$ extract, was not found in the distilled oil.

The composition of the commercial Artemisia essential oils samples is displayed in Table 2. In contrast to the own preparations of $A$. abrotanum oils, the commercial sample was dominated by the sesquiterpene silphiperfolene derivatives and contained appreciable amounts of 1,8-cineol $(21.7 \%, 168 \mathrm{mg} / \mathrm{ml})$, borneol $(8.2 \%, 64 \mathrm{mg} / \mathrm{ml})$ and $p$-cymene $(6.9 \%, 54 \mathrm{mg} / \mathrm{ml})$. The $A$. dracunculus sample had estragol as main compound and the ocimene isomers similar to the microdistillation fingerprint of the own material from the botanical garden. The commercial A. absinthium sample had $\beta$-thujone $(41 \%, 358 \mathrm{mg} / \mathrm{g})$, sabinyl acetate $(22 \%, 153 \mathrm{mg} / \mathrm{g})$ and $\alpha$-thujone $(13 \%, 113 \mathrm{mg} / \mathrm{ml})$ as main compounds. Finally, the purchased A. vulgaris essential oil was rich in $\alpha$-thujone $(65 \%, 531 \mathrm{mg} / \mathrm{g})$, camphor $(14 \%$, $110 \mathrm{mg} / \mathrm{g})$, and $\beta$-thujone $(10 \%, 79 \mathrm{mg} / \mathrm{g})$.

\section{Test microorganisms}

The anticandidal test with the commercial oils is presented in Table 3 . All four oils showed anticandidal activity with $A$. abrotanum being the most efficient, followed by A. absinthium, A. dracunculus and A. vulgaris.

The four investigated Artemisia species revealed distinct volatile patterns. A comparison with published data showed that even within one and the same species the oil composition may vary widely, representing different chemotypes. For instance, A. abrotanum from Cuba had trans-sabinyl acetate and $\alpha$-terpineol as main oil compounds [12]. The same species collected in Serbia displayed silphiperfol-5-en3-one A (14.6\%), ascaridole (13.1\%), 1,8-cineole (10.5\%), $\alpha$ bisabolol oxide A acetate (8.7\%) as main oil components [13]. Similarly, an oil from Northwestern Italy had 1,8-cineole (34.7\%), bisabolol oxide (18.4\%) and ascaridole (16.0\%) as main compounds [14]. The dominant components in the oil from the Crimea were 1,8-cineole and camphor [15]. A German A. abrotanum showed 1,8-cineol as main oil compound [16]. Plant cultivated in Poland were rich in piperitone (17.5\%), davanone (16.8\%), 1,8-cineole (12.5\%) and silphiperfol-5-en-3-ol A (6.3\%). These plants contained also the artedouglasia oxides as minor compounds [17].

The present oil of $A$. dracunculus and the oils reported in literature were dominated by phenylpropanoids. A Polish oil sample contained elemicin (48.8\%), sabinene (18.9\%), isoelemicin (13.3\%) and eugenol (7.6\%) [17], different Finnish French tarragon contained cis- and trans-ocimenes, estragole and herniarin [18], and Crimean samples sabinene and elemicine [19]. Canadian A. dracunculus essential oil was mainly composed of methyl eugenol (35.8\%), eugenol (16.2\%) and terpinolene (19.1\%) [20]. 
Table 1 GS-MS chemical composition of the volatiles from the investigated Artemisia species

\begin{tabular}{|c|c|c|c|c|c|c|c|c|c|}
\hline No & RI & Compound & $\begin{array}{c}\text { A. abrotanum } \\
\text { plant } \%(\mathrm{mg} / \mathrm{ml})\end{array}$ & $\begin{array}{c}\text { A. abrotanum } \\
\mathrm{CH}_{2} \mathrm{Cl}_{2} \%(\mathrm{mg} / \mathrm{ml})\end{array}$ & $\begin{array}{l}\text { A. dracunculus } \\
\text { plant } \%(\mathrm{mg} / \mathrm{ml})\end{array}$ & $\begin{array}{l}\text { A. dracunculus } \\
\mathrm{CH}_{2} \mathrm{Cl}_{2} \%(\mathrm{mg} / \mathrm{ml})\end{array}$ & $\begin{array}{c}\text { A. absinthium } \\
\mathrm{CH}_{2} \mathrm{Cl}_{2} \%(\mathrm{mg} / \mathrm{ml})\end{array}$ & $\begin{array}{c}\text { A. vulgaris } \\
\text { plant } \%(\mathrm{mg} / \mathrm{ml})\end{array}$ & $\begin{array}{l}\text { A. vulgaris } \% \\
(\mathrm{mg} / \mathrm{ml})\end{array}$ \\
\hline 1. & 969 & 4-Methyl-pent-2-enolid & $15.7(1.7869)$ & $1.7(0.1379)$ & - & $1.8(0.046)$ & - & - & - \\
\hline 2. & 991 & 1-Octen-3ol & - & - & - & - & - & - & $4.2(0.253)$ \\
\hline 3. & 1037 & 1,8-Cineol & - & - & - & - & - & - & $18(1.077)$ \\
\hline 4. & 1043 & Z- $\beta$-Ocimene & - & - & $1.3(0.103)$ & - & - & - & - \\
\hline 5. & 1044 & Lavender lactone & $2.6(0.3017)$ & - & - & - & - & - & - \\
\hline 6. & 1053 & E- $\beta$-Ocimene & - & - & $1.6(0.124)$ & - & - & - & - \\
\hline 7. & 1069 & cis-Arbusculone & $0.7(0.0885)$ & - & - & - & - & - & - \\
\hline 8. & 1077 & trans-Arbusculone & $0.6(0.0738)$ & - & - & - & - & - & - \\
\hline 9. & 1100 & trans-Sabinene hydrate & - & - & - & - & - & $3.5(0.079)$ & - \\
\hline 10. & 1114 & Phenylethyl alcohol & - & - & - & - & - & $1.8(0.042)$ & $2.1(0.129)$ \\
\hline 11. & 1122 & $\beta$-Thujone & - & - & - & - & $14.7(0.380)$ & - & - \\
\hline 12. & 1129 & 1-Terpineol & $1.6(0.1817)$ & - & - & - & - & - & - \\
\hline 13. & 1132 & allo Ocimene & - & - & $0.3(0.028)$ & - & - & - & - \\
\hline 14. & 1141 & iso-3-Thujanol & - & - & - & - & $2.2(0.059)$ & - & - \\
\hline 15. & 1147 & cis- $\beta$-Terpineol & $1.2(0.1402)$ & $0.8(0.0700)$ & - & - & - & - & - \\
\hline 16. & 1152 & Camphor & - & - & - & - & - & - & $5.6(0.337)$ \\
\hline 17. & 1173 & Borneol & - & - & - & - & - & $5.4(0.122)$ & $13.2(0.792)$ \\
\hline 18. & 1183 & Terpinen-4-ol & - & - & - & - & - & $18.2(0.407)$ & $13.5(0.801)$ \\
\hline 19. & 1189 & p-Cymen-8-ol & - & - & - & - & - & - & $1.9(0.113)$ \\
\hline 20. & 1195 & a-Terpineol & - & - & - & - & - & $10(0.223)$ & $6.6(0.394)$ \\
\hline 21. & 1202 & Estragole & $0.9(0.1123)$ & $0.8(0.0702)$ & $84.1(6.406)$ & - & $5(0.126)$ & - & \\
\hline 22. & 1211 & trans-Piperitol & $1.2(0.1454)$ & - & - & - & - & - & - \\
\hline 23. & 1221 & trans-Carveole & - & - & - & - & - & $1.9(0.043)$ & - \\
\hline 24. & 1226 & Nerol & - & - & - & - & $11.5(0.297)$ & - & - \\
\hline 25. & 1227 & Nordavanone, & $3(0.3414)$ & $5.4(0.4275)$ & - & $8.3(0.206)$ & - & - & - \\
\hline 26. & 1252 & Geraniol & - & - & - & - & $2.6(0.069)$ & - & - \\
\hline 27. & 1253 & Chavicol & - & & $0.4(0.031)$ & $1(0.023)$ & - & - & - \\
\hline 28. & 1257 & Piperitone & $0.5(0.0616)$ & - & - & - & - & - & - \\
\hline 29. & 1260 & cis-Chrysanthenylacetate & - & - & - & - & $2.5(0.065)$ & - & $15(0.897)$ \\
\hline 30. & 1292 & Cuminol & - & - & - & - & & $7.2(0.163)$ & $1.7(0.105)$ \\
\hline 31. & 1299 & UnknownABS & & & & & $29.1(0.750)$ & & \\
\hline 32. & 1331 & 1.4-p-Menthadien-7-ol & - & - & - & - & & $3.6(0.082)$ & $1.7(0.105)$ \\
\hline
\end{tabular}


Table 1 GS-MS chemical composition of the volatiles from the investigated Artemisia species (Continued)

\begin{tabular}{|c|c|c|c|c|c|c|c|c|c|}
\hline 33. & 1354 & Eugenol & - & & $1.4(0.107)$ & $1.4(0.036)$ & $0.9(0.023)$ & $3.8(0.086)$ & $3(0.175)$ \\
\hline 34. & 1367 & UnknownABR1 & & $5.2(0.4115)$ & - & - & - & - & - \\
\hline 35. & 1386 & E-Methylcinnamate & - & - & $0.9(0.068)$ & - & - & - & - \\
\hline 36. & 1399 & Methyleugenol & - & & $2.2(0.173)$ & $1.4(0.035)$ & $1.5(0.040)$ & - & - \\
\hline 37. & 1401 & UnknownABR2 & $1.3(0.1540)$ & & & & & & \\
\hline 38. & 1422 & UnknownABR3 & & $20.7(1.6363)$ & & $4.3(0.106)$ & & & \\
\hline 39. & 1466 & Y-Decalactone & - & - & $0.2(0.015)$ & - & - & - & - \\
\hline 40. & 1483 & Germacrene D & - & - & $0.2(0.015)$ & - & - & $0.5(0.011)$ & $1.6(0.091)$ \\
\hline 41. & 1484 & Davanone ether & $0.9(0.1016)$ & - & - & - & - & - & \\
\hline 42. & 1486 & Farnesene & - & - & - & - & - & - & $1(0.062)$ \\
\hline 43. & 1491 & $\beta$-Selinene & - & - & - & - & - & - & $1.2(0.076)$ \\
\hline 44. & 1500 & Bicyclogermacrene & - & - & $0.1(0.012)$ & - & - & - & - \\
\hline 45. & 1504 & Davana ether* & $3.2(0.3591)$ & - & - & - & - & - & - \\
\hline 46. & 1518 & Artedouglasia oxide C & $1.7(0.1932)$ & $1.1(0.0869)$ & & $1.7(0.042)$ & & & \\
\hline 47. & 1520 & S-Cadinene & - & - & - & - & - & - & $1.5(0.093)$ \\
\hline 48. & 1522 & Davana ether* & $1.4(0.1581)$ & - & - & - & - & - & - \\
\hline 49. & 1531 & Artedouglasia oxide A & $2.2(0.2575)$ & $1.8(0.1486)$ & - & $3.4(0.084)$ & - & - & - \\
\hline 50. & 1554 & Artedouglasia oxide D & $1(0.1201)$ & $0.6(0.0534)$ & - & $1(0.023)$ & - & - & - \\
\hline 51. & 1476 & Artedouglasia oxide B & $1.3(0.1469)$ & $1(0.0858)$ & - & $1.6(0.040)$ & - & - & - \\
\hline 52. & 1580 & cis-Davanone & $5.2(0.5989)$ & $7.4(0.5896)$ & $0.6(0.048)$ & $9.1(0.225)$ & - & - & - \\
\hline 53. & 1589 & Spathulenol & - & - & - & - & $6.5(0.168)$ & $12.1(0.271)$ & $7.1(0.425)$ \\
\hline 54. & 1585 & Caryophyllene oxide & - & - & - & - & $2.8(0.072)$ & 8.5 (0.191) & $2.7(0.163)$ \\
\hline 55. & 1598 & UnknownABR4 & - & $2(0.1582)$ & - & - & - & - & - \\
\hline 56. & 1599 & UnknownABR5 & $1.3(0.1570)$ & - & - & - & - & - & - \\
\hline 57. & 1641 & UnknownABR6 & $2.7(0.3121)$ & - & - & - & - & - & - \\
\hline 58. & 1655 & Bisabololoxide B & - & - & - & - & $3.3(0.086)$ & - & - \\
\hline 59. & 1656 & epi-a Muurolol & - & - & - & - & - & $11.8(0.265)$ & - \\
\hline 60. & 1684 & a-Bisabolol & - & - & - & & $11(0.285)$ & - & - \\
\hline 61. & 1685 & UnknownABR7 & $12.4(1.4113)$ & $17.2(1.3599)$ & $0.5(0.041)$ & $20.5(0.506)$ & - & - & - \\
\hline 62. & 1688 & UnknownABR8 & $4(0.4598)$ & - & - & - & - & - & - \\
\hline 63. & 1711 & UnknownABR9 & $8.7(0.9892)$ & - & - & $3.4(0.085)$ & - & - & - \\
\hline 64. & 1719 & Herniarin, & - & - & $4.3(0.329)$ & $10(0.246)$ & $5.8(0.150)$ & - & - \\
\hline 65. & 1754 & Davanone Derivative & $22.5(2.5593)$ & $33.3(2.6535)$ & - & $18.2(0.450)$ & - & - & - \\
\hline
\end{tabular}


Table 1 GS-MS chemical composition of the volatiles from the investigated Artemisia species (Continued)

\begin{tabular}{lllccccc}
\hline 66. & 1936 & UnknownDRA & - & - & $0.3(0.029)$ & $7(0.174)$ & - \\
67. & 1963 & Hexadecanoic acid & - & - & - & - & - \\
68. & 2107 & Phytol-isomer & $1.2(0.1451)$ & - & $1.1(0.087)$ & $(0.138)$ & - \\
\hline
\end{tabular}

\section{* exact isomer not determined, RI retention index}

Mass spectra of the unidentified compounds.

Rl 1367 UnknownABR1: 41 (50), 43 (72), 44 (13), 45 (14), 53 (15), 55 (100), 56 (13), 57 (30), 67 (32), 68 (13), 69 (37), 71 (41), 74 (11), 81 (19), $83(14), 84(11), 85$ (11), 93 (37), 95 (25), 111 (34), 123 (10), 169 (46),

RI 1299 UnknownABS: 41 (39), 43 (68), 44 (8), 53 (16), 55 (22), 57 (14), 59 (100), 67 (49), 68 (19), 69 (9), $71(34), 77$ (11), $79(32), 81(45), 82(55), 83(12), 89(11), 93(7), 94$ (7), 109 (10), $152(11)$.

(1)

RI 1422 UnknownABR3: 41 (32), 43 (62), 53 (13), 55 (83), 57 (10), 67 (24), 68 (14), 69 (28), 71 (100), $72(14), 77$ (10), 81 (24), $83(16), 91$ (14), 93 (74), 95 (9), 100 (19), $110(11), 111$ (94), 125 (18), 195 (11).

RI 1598 UnknownABRA: 41 (9), 42 (3), $43(10), 44(3), 53(4), 55(13), 57(6), 67(5), 68(3), 69(5), 71(4), 79(2), 81(4), 83(5), 85(100), 86(5), 93(9), 107(2), 111(3), 114(12), 156(4)$,

RI 1599 UnknownABR5: 41 (38), $43(100), 44(14), 55(30), 57(25), 69(22), 71(17), 77(14), 79(20), 81(32), 83(22), 85(20), 91(15), 93(18), 95(17), 109(45), 149(14), 161(15), 207(46), 219(21), 234(15)$,

RI 1641 UnknownABR: 41 (52), $43(68), 44(13), 53(18), 55(100), 56(13), 57(29), 67(27), 68(18), 69(37), 71(23), 79(12), 81(27), 83(32), 84(17), 85(12), 91(12), 93(72), 95(24), 109(13), 111(73)$.

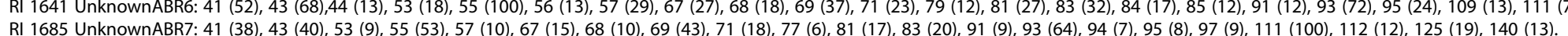

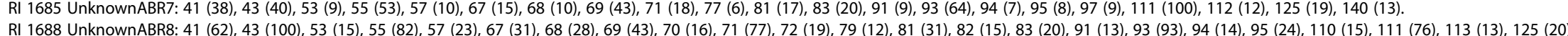

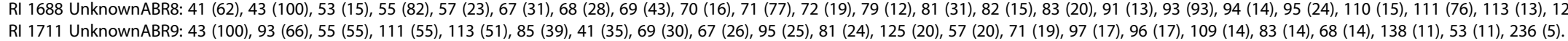

RI 1754 Davanone-derivative: 41 (45). 43 (100), 53 (17), 55 (74), 57 (24), 67 (32), 68 (14), 69 (45), 71 (36), 79 (18), 81 (48), 82 (12), 83 (23), 91 (12), $93(87), 95(20), 96(29), 97$ (48), 109 (20), 111 (90), 112 (12), 113 (15), 125

(69), 236 (36).

Rl 136 UnknownDRA: 41 (40), 43 (12), 53 (22), 55 (23), 57 (17), 66 (23), 67 (28), 69 (25), 77 (12), 79 (18), 81 (84), 82 (9), 83 (12), 95 (21), 96 (74), 98 (14), 110 (17), 113 (13), 151 (100), 152 (37), 22. 
Table 2 Composition of the commercial Artemisia essential oils ( $\mathrm{mg} / \mathrm{ml}$ )

\begin{tabular}{|c|c|c|c|c|c|c|}
\hline No & RI & Compound & $\begin{array}{l}\text { A. abrotanum } \% \\
(\mathrm{mg} / \mathrm{ml})\end{array}$ & $\begin{array}{c}\text { A. dracunculus \% } \\
(\mathrm{mg} / \mathrm{ml})\end{array}$ & $\begin{array}{c}\text { A. absinthium } \% \\
(\mathrm{mg} / \mathrm{ml})\end{array}$ & $\begin{array}{l}\text { A. vulgaris \% } \\
(\mathrm{mg} / \mathrm{ml})\end{array}$ \\
\hline 1 & 800 & Octane & - & $0.1(0.8)$ & 01. (0.9) & \\
\hline 2 & 927 & Tricyclene & $0.08(0.6)$ & & & $0.1(0.8)$ \\
\hline 3 & 932 & a-Thujene & - & & & $0.03(0.3)$ \\
\hline 4 & 939 & a-Pinene & $0.1(1.1)$ & $1.4(11.9)$ & $0.3(2.9)$ & $0.1(1.0)$ \\
\hline 5 & 953 & Camphene & $2.7(18.5)$ & $0.05(0.5)$ & $0.1(1.2)$ & $2.2(18.0)$ \\
\hline 6 & 978 & Sabinene & $0.1(0.9)$ & $0.07(0.6)$ & $2(16.1)$ & $1.8(15.2)$ \\
\hline 7 & 982 & $\beta$-Pinene & $0.3(2.3)$ & $0.1(1.5)$ & $0.3(2.6)$ & $0.2(1.6)$ \\
\hline 8 & 993 & Myrcene & & $0.1(0.9)$ & $6.4(6.5)$ & \\
\hline 9 & 1008 & a-Phellandrene & & & $0.2(1.6)$ & \\
\hline 10 & 1021 & a-Terpinene & $0.9(6.4)$ & & & \\
\hline 11 & 1030 & p-Cymene & $7.8(53.5)$ & & $0.1(1.4)$ & $0.7(6.3)$ \\
\hline 12 & 1034 & Limonene & & $4(33.8)$ & $0.4(3.4)$ & \\
\hline 13 & 1037 & 1,8-Cineol & $24.5(167.9)$ & & $1.5(12.3)$ & $0.6(5.1)$ \\
\hline 14 & 1042 & $Z$ - $\beta$-Ocimene & & $8.3(69.5)$ & & \\
\hline 15 & 1053 & E- $\beta$-Ocimene & & $7.8(65.5)$ & & \\
\hline 16 & 1064 & $Y$-Terpinene & $0.4(3.1)$ & & $0.1(1.0)$ & \\
\hline 17 & 1073 & cis-Sabinene hydrate & $0.3(2.0)$ & & & $0.02(0.2)$ \\
\hline 18 & 1092 & Terpinolene & $0.1(0.7)$ & & & \\
\hline 19 & 1093 & Fenchone & & $0.3(3.0)$ & & \\
\hline 20 & 1102 & Linalool & & & $2(16.4)$ & \\
\hline 21 & 1102 & trans-Sabinene hydrate & $0.3(2.4)$ & & & $0.07(0.6)$ \\
\hline 22 & 1110 & a-Thujone & & & 13. (112.5) & $65.5(530.5)$ \\
\hline 23 & 1122 & $\beta$-Thujone & & & $44(357.6)$ & $9(73.2)$ \\
\hline 24 & 1126 & Dehydro sabinaketone & & & & $1.5(12.8)$ \\
\hline 25 & 1132 & allo-Ocimene & & $0.1(1.0)$ & & \\
\hline 26 & 1136 & Z-Epoxyocimene & & & $1.5(12.9)$ & \\
\hline 27 & 1146 & trans-Sabinol & & & $3(24.2)$ & \\
\hline 28 & 1146 & trans-Pinocarveol & & & & $0.5(4.1)$ \\
\hline 29 & 1146 & neo-allo-Ocimene & & $0.07(0.6)$ & & \\
\hline 30 & 1152 & Camphor & $3.5(24.2)$ & & $0.8(7.2)$ & $13.6(110.4)$ \\
\hline 31 & 1165 & Sabina ketone & & & & $0.3(2.4)$ \\
\hline 32 & 1171 & Isoborneol & & & $0.8(6.6)$ & \\
\hline 33 & 1173 & Borneol & $9.3(63.8)$ & & & $0.4(3.9)$ \\
\hline 34 & 1183 & Terpinen-4-ol & $1.8(12.7)$ & & $0.3(2.9)$ & $0.5(4.4)$ \\
\hline 35 & 1195 & a-Terpineol & $0.2(1.5)$ & & & \\
\hline 36 & 1201 & Myrtenal & $0.37(2.6)$ & & & $0.4(3.2)$ \\
\hline 37 & 1202 & Estragol & & $75(626.1)$ & & \\
\hline 38 & 1235 & Isobornylformate & $0.1(1.3)$ & & & \\
\hline 39 & 1240 & Fenchyl acetate & & $0.1(0.8)$ & & \\
\hline 40 & 1247 & trans-Chrysanthenyl acetate & $1(7.2)$ & & & \\
\hline 41 & 1248 & Cuminal & & & & $0.1(1.0)$ \\
\hline 42 & 1257 & Piperitone & & & & $0.2(1.6)$ \\
\hline
\end{tabular}


Table 2 Composition of the commercial Artemisia essential oils (mg/ml) (Continued)

\begin{tabular}{|c|c|c|c|c|c|c|}
\hline 43 & 1266 & Verbenyl acetate & & & & $0.4(3.4)$ \\
\hline 44 & 1291 & Bornyl acetate & $0.7(5.2)$ & & & $0.07(0.6)$ \\
\hline 45 & 1291 & Anethol & & $1.5(12.7)$ & & \\
\hline 46 & 1292 & Lavandulyl acetate & & & $0.4(3.7)$ & \\
\hline 47 & 1296 & Sabinyl acetate & & & $23.7(193.2)$ & \\
\hline 48 & 1334 & Silphiperfol-5-ene & $0.7(5.3)$ & & & \\
\hline 49 & 1346 & Silphiperfolen-Isomer & $0.5(3.9)$ & & & \\
\hline 50 & 1353 & $7 \beta$-H-Silphiperfol-5-ene & $0.6(4.4)$ & & & \\
\hline 51 & 1367 & Silphiperfol-4, 7(14)-diene & $0.3(2.6)$ & & & \\
\hline 52 & 1369 & Silphiperfolen-Isomer & $0.7(5.0)$ & & & \\
\hline 53 & 1384 & a-Copaene & & & $0.1(1.4)$ & $0.07(0.6)$ \\
\hline 54 & 1408 & Z-Methyl-isoeugenol & & $0.2(2.2)$ & & \\
\hline 55 & 1425 & Linalyl isobutyrate & & & $0.2(1.7)$ & \\
\hline 56 & 1429 & $\beta$-Caryophyllene & $0.7(5.3)$ & $0.05(0.5)$ & $1.4(11.8)$ & \\
\hline 57 & 1464 & a-Humulene & $1.8(1.3)$ & & $0.1(1.1)$ & \\
\hline 58 & 1483 & Germacrene D & $3.4(23.5)$ & $0.05(0.5)$ & & \\
\hline 59 & 1497 & Farnesene & & $0.04(0.4)$ & & \\
\hline 60 & 1500 & Bicyclogermacrene & & $0.05(0.5)$ & $0.3(2.4)$ & \\
\hline 61 & 1507 & Farnesene & & & & $0.03(0.3)$ \\
\hline 62 & 1512 & Lavandulyl isovalerate & & & $0.7(5.9)$ & \\
\hline 63 & 1513 & Unknown & $5.7(39.4)$ & & & \\
\hline 64 & 1531 & $\beta$-Sesquiphellandrene & & $0.05(0.5)$ & & \\
\hline 65 & 1531 & Unknown & $1.7(12.1)$ & $0.1(0.9)$ & & \\
\hline 66 & 1548 & Siphiperfol-5-en-3-one B & $2.7(19.1)$ & & & \\
\hline 67 & 1558 & Silphiperfol-5-en-3-one B-isomer & $5.1(35.2)$ & & & \\
\hline 68 & 1583 & Silphiperfol-5-en-3-one A & $18.9(129.5)$ & & & \\
\hline 69 & 1589 & Spathulenol & & & & \\
\hline 70 & 1595 & Unknown & $3(20.1)$ & & & \\
\hline
\end{tabular}

According to the literature [21], the essential oil composition of $A$. absinthium L. was obtained and examined from different geographical parts of Europe the Absithii herba (plant) can be divided into at least 4 chemotypes: thujones rich oil (Greece, Spain, Ukraine, France, Italy), sabinene acetate rich oil (Armenia, Latvia, Belgium, Lithuania), epoxyocimenes rich oil (Russia), and a chemotype in which oil

Table 3 Inhibitory in vitro effects of the commercial Artemisia essential oils on Candida albicans (ATCC 10231)

\begin{tabular}{ll}
\hline Essential oil & $\begin{array}{l}\text { Inhibition zone } \\
\text { diameter }(\mathbf{m m} \pm \mathbf{S D})\end{array}$ \\
\hline A. dracunculus & $15.5 \pm 2.1$ \\
A. abrotanum & $20.0 \pm 1.4$ \\
A. absinthium & $17.0 \pm 1.4$ \\
A. vulgaris & $12.5 \pm 0.7$ \\
Nystatin (positive control) & $15.0 \pm 0$ \\
Blank filter paper (negative control) & $6.0 \pm 0$ \\
\hline
\end{tabular}

monoterpenes sabinene and myrcene were predominant (Estonia, Scotland, Moldova, Hungary). This is partly in accordance with a former study [22] where 19 samples of $A$. absinthium from Italy, France, Romania, and Siberia were divided into four chemotypes: sabinyl acetate rich oil, epoxyocimenes rich oil, chrysanthenyl acetate rich oil, and thujone rich oil. An epoxy-ocimene rich oil was also found in northwestern Italy [14]. An oil rich in t-sabinylacetate, thujone and myrcene is reported from Canada [20]. Furthermore a Turkish $A$. absinthium oil had chamazulene and nuciferol esters as major compounds [23].

Cited literature $[5,15]$ described an $A$. absinthium oil rich in thujone and in the aerial parts of A.vulgaris an oil with high proportions of 1,8-cineole, sabinene, thujone, and caryophyllene oxide.

Essential oils from $A$. vulgaris rich in camphor, 1,8 cineole or $\beta$-thujone have been described [14,24-26]. These oils contain also chrysanthenyl acetate, borneol, methyleugenol, $\alpha$-terpineol, $t$-verbenol, or $\beta$ caryophyllene but owing to the 
great variability present it is difficult to point out distinct chemotypes. The A. vulgaris volatile fraction in the present study contained terpinen-4-ol, borneol, cis-chrysanthenyl acetate, and spathulenol as major compounds.

In this study, all volatile fractions isolated from all four Artemisia species showed some activity against Candida albicans. The highest activity was found for A. abrotanum where an inhibition zone larger than that produced by $\mathrm{Ny}$ statin could be observed. But it has to be considered that these fractions contain higher amounts of potentially active compounds than the fractions obtained from the other investigated species. Nevertheless, there are reports confirming the antimycotic activity of the main compounds identified by GC-MS in the present volatile fractions.

An extract from A. abrotanum grown in Sweden as well as the component davanone were effective against $C$. albicans [27]. Furthermore, davana oil from Artemisia pallens rich in davanone and the davana fraction derived from this oil were active against Candida albicans [28]. However in another study, davanone as single compound showed only very weak in vitro activity against C. albicans [29]. Candida albicans proved to be very susceptible against the essential oil from Ocimum selloi which contains more than 95\% estragole (= methylchavicol) [30]. However, in another test series, estragole as single substance proved to be ineffective [31].

In the present study, 1,8-cineol, borneol, terpinen-4-ol, spathulenol, $\beta$-thujone, and $\alpha$-bisabolol were major compounds in all of the volatile fractions of $A$. absinthium and A. vulgaris studied. The oils containing these compounds can be found in various plants and some of them were reported to inhibit the growth of $C$. albicans. An essential oil rich in $\alpha$-pinene and $\alpha$-bisabolol from Laserpitium zernyi showed a low activity against two strains of C. albicans [32]. The oil of $A$. annua containing 48\% camphor as main compound was active against $C$. albicans [33]. A sage (Salvia officinalis) oil from Montenegro with the main compounds $\alpha$-thujone (29.5\%), camphor (22.5\%) and 1,8-cineole (12.2\%) had significant activity against C. albicans [34].

Oils from sage, myrtle, and laurel containing 1,8-cineol as main compound showed some activity against C. albicans [35], also an Eucalyptus oil with $85 \%$ of 1,8-cineole content [36]. However an Egyptian oil from Eucalyptus occidentalis that presumably contained 1,8 cineole had no influence on the growth of C. albicans [37].

Additionally, 7-methoxycoumarin (herniarin) isolated from twigs of Treculia obovoidea showed some activity against various Candida species [38]. Although reports on the antifungal activity of Artemisia species are inconsistent the results of this study confirm that Artemisia essential oils exert antimycotic effects and may represent good candidates to replace in the future allopathic treatments to which Candida has developed resistance. Under the conditions of this study, volatile fractions from $A$. abrotanum as well as the commercial oil from this plant had the strongest effect on Candida albicans although they differed in composition, having either davanone derivatives or silphiperfolene derivatives and 1,8-cineol as major compounds, respectively. The differences in activity and order in efficacy of the oils and volatile preparations most probably arises from differences in composition. So the own A. vulgaris preparations containing a mix of 1,8-cineol, terpinen-4-ol, borneol, camphor, and spathulenol were more active than the commercial oil with the thujones and camphor. The lower activity of the A. absinthium dichloromethane extract might be due to the lower overall concentration of active compounds in this preparation as compared to the commercial sample from the same species.

\section{Experimental research \\ Plant material}

The A. abrotanum, A. dracunculus, and A. absinthium aerial parts were collected in the Botanical Garden of the University of Veterinary Medicine Vienna, Austria. A. vulgaris aerial parts were gathered in the surroundings of Timisoara (Bencecul de Sus), Romania. All plants were picked during the June-September period of 2010, while blooming. Voucher specimen of the collected plants were deposited in the Herbarium of the University of Vienna (WU-Generale, http:\\herbarium.univie.ac.at).

\section{Commercial essential oils}

Additionally, other commercial essential oils from Artemisia absinthium (USA), A. dracunculus (Iran), and $A$. vulgaris (Marokko) were obtained from Baccara Rose (Dagmar Köhler, 47665 Sonsbeck, Germany). Artemisia abrotanum came from Ayus GmbH, 77815 Bühl/Moos, Germany.

\section{Test microorganisms}

Isolates of Candida albicans (ATCC 10231) were obtained from the culture collection of the Department of Microbiology, Banat's University of Agricultural Sciences and Veterinary Medicine Timisoara.

\section{Methods}

The preparation of the essential oils was done using a modified standard procedure according to the European Pharmacopoeia [39]. The procedures were done at the same period of time. The plant material (15 g) was subjected to hydrodistillation using $150 \mathrm{ml}$ distilled water for 4 hours at a constant rate. An average of $18.5 \mathrm{mg}$ of essential oil was obtained.

In addition, an extraction with dichloromethane $\left(\mathrm{CH}_{2} \mathrm{Cl}_{2}\right)$ followed by hydrodistillation was carried out. $100 \mathrm{~g}$ of fresh plant material (leaves or flowers) were extracted 5 times with $100 \mathrm{ml}$ of $\mathrm{CH}_{2} \mathrm{Cl}_{2}$ in consecutive steps. The resulting extracts were combined and the 
solvent was removed in vacuo using a Rotavapor. The average yield was $2 \mathrm{~g}$ of crude extract; the pooled extract was hydrodistillated for 3 hours as mentioned above and stored at $4^{\circ} \mathrm{C}$ until analysis. An average yield of $30 \mathrm{mg}$ oil out of $2 \mathrm{~g}$ extract was obtained. For further analyses volatile oil from several preparations were combined.

Leaves of the plants collected from the botanical garden were also subjected to microdistillation using the automatic microdistillation unit MicroDistiller from Eppendorf (Hamburg, Germany) which is a gentle distillation method that allows investigating essential oil finger prints. The procedure was as follows: 0.2 to $0.3 \mathrm{~g}$ finely crushed dried plant material and $10 \mathrm{ml}$ distilled water were filled into the sample vial. The collecting vial containing $1 \mathrm{ml}$ water, $0.5 \mathrm{~g} \mathrm{NaCl}$ and $300 \mu \mathrm{l} n$-hexane was connected with a capillary to the sample vial. The heating program applied to the sample vial was $15 \mathrm{~min}$ at $108^{\circ} \mathrm{C}$ followed by $45 \mathrm{~min}$ at $112^{\circ} \mathrm{C}$. The collecting vial was kept at $-2^{\circ} \mathrm{C}$, where the volatiles were trapped in $0.3 \mathrm{ml} n$-hexane.

\section{Analysis of the essential oils}

The analysis of the volatiles was performed using a Hewlett-Packard 6890 GC linked to a Hewlett- Packard 5973 mass-selective detector. For the analysis a Zebron ZB$5 \mathrm{MS}$, capillary column $(27 \mathrm{~m} \times 250 \mu \mathrm{m}$ i. d., $0.25 \mu \mathrm{m}$ film thickness) was used. The carrier gas was helium at $1.3 \mathrm{ml} /$ min in constant flow mode. The injector temperature was $250^{\circ} \mathrm{C}$, the injection volume $1 \mu \mathrm{l}$, and the split ratio 1:20. The initial oven temperature of $60^{\circ} \mathrm{C}$ was held for 1 minute, then increased at a rate of $5^{\circ} \mathrm{C} / \mathrm{min}$ up to $220^{\circ} \mathrm{C}$, and subsequently at a rate of $15^{\circ} \mathrm{C} / \mathrm{min}$ up to $280^{\circ} \mathrm{C}$, and finally was held isothermal for $1 \mathrm{~min}$. The transfer line to the MSD was set at $280^{\circ} \mathrm{C}$ and the scan conditions were: $\mathrm{M} / \mathrm{Z} 40-$ 300 , at 1.75 scans/sec.

Prior to analysis $900 \mu \mathrm{l}$ of the volatile fractions were mixed with $100 \mu \mathrm{l}$ of biphenyl $(2.0 \mathrm{mg} / \mathrm{ml}$ in hexane) as internal standard. The components of essential oils were identified by comparing their relative retention times and mass spectra with those of Registry of Mass Spectral Data and literature citations $[40,41]$. The amount of the individual compounds in the fractions was calculated using the Total Ion Current from the MSD signal and assuming the same response as for the internal standard biphenyl.

Inocula containing $10^{6}$ cells $/ \mathrm{ml}$ were spread on the medium (Sabouraud with added penicillin (40 units $/ \mathrm{ml}$ ) and streptomycin $(2 \mathrm{mg} / \mathrm{ml}$ of medium $)$ ). The antifungal activity test was carried out by the disk diffusion method using sterilized $6 \mathrm{~mm}$ diameter filter paper disks. From each volatile oil preparation a quantity of $50 \mu \mathrm{l}$ was submitted to testing and the tests were done in triplicate. The inoculated plates were incubated at $35^{\circ} \mathrm{C}$ for $24 \mathrm{~h}$. Standard bio discs of Nystatin 100 units/disk (Himedia Laboratories Ltd., India) were used as positive control and blank sterilized filter papers as negative control.
After the incubation, the diameter of the inhibition zone for each essential oil was measured in millimeters (including the $6 \mathrm{~mm}$ diameter of the disk). The results are expressed as mean values of three determinations \pm S.D.

\section{Antifungal activity in vitro}

The experimental protocols were approved by the Animal Ethics Committee of the Faculty of Veterinary Medicine Timisoara and conducted accordingly. All 7 Artemisia preparations were subjected to the disc diffusion test against Candida albicans (ATCC 10231). Inhibition zones observed ranged from $23.5 \mathrm{~mm}$ to $13 \mathrm{~mm}$ for the essential oils and volatile oils. An $20.5 \mathrm{~mm}$ inhibition zone was determined for Nystatin and $6 \mathrm{~mm}$ (the diameter of the biodisc) for the negative control (Table 4).

Highest antifungal activity was observed with $A$. abrotanum (both volatile fractions) followed by $A$. vulgaris, A. dracunculus, and A. absinthium.

\section{Conclusions}

Since all volatile fractions caused inhibition we conclude that compounds found in different quantities are responsible for the varying in vitro-activity against $C$. albicans, but it is difficult to attribute this effect of complex mixtures to a particular constituent. Possibly synergistic as well as antagonistic effects of compounds in the oil should also be taken into consideration as often stronger antifungal activity can be observed with complete essential oils in comparison to single oil components [42]. Our studies showed that different procedures for preparation of volatile fractions, starting from extracts or plant material, resulted in different chemical compositions. This phenomenon may contribute to the contradictory results published for the antimycotic effects of Artemisia. Under the conditions of this study, volatile fractions from Artemisia plants exert antifungal in vitro activity. Our results may open paths for the development of new phytotherapeutic products from the Artemisia species studied.

Table 4 Inhibitory in vitro effects of the volatile fractions on Candida albicans (ATCC 10231)

\begin{tabular}{ll}
\hline Volatile fraction & $\begin{array}{l}\text { Inhibition zone } \\
\text { diameter }(\mathbf{m m} \pm \mathbf{S D} \text { ) }\end{array}$ \\
\hline A. dracunculus (oil) & $15.5 \pm 1.52$ \\
A. dracunculus $\left(\mathrm{CH}_{2} \mathrm{Cl}_{2}\right)$ & $13.0 \pm 2.08$ \\
A. abrotanum (oil) & $23.5 \pm 1.52$ \\
A. abrotanum $\left(\mathrm{CH}_{2} \mathrm{Cl}_{2}\right)$ & $21.5 \pm 3.51$ \\
A. absinthium $\left(\mathrm{CH}_{2} \mathrm{Cl}_{2}\right)$ & $13.0 \pm 1.15$ \\
A. vulgaris (oil) & $16.6 \pm 1.57$ \\
A. vulgaris $\left(\mathrm{CH}_{2} \mathrm{Cl}_{2}\right)$ & $17.0 \pm 1.15$ \\
Nystatin (positive control) & $20.5 \pm 4.04$ \\
Blank filter paper (negative control) & $6.0 \pm 0$ \\
\hline
\end{tabular}




\author{
Abbreviations \\ C. albicans: Candida albicans; GC-MS: Gas chromatography-mass \\ spectrometry.
}

\section{Competing interests}

The authors declare that they have no competing interests.

\section{Authors' contributions}

$\mathrm{OD}, \mathrm{SI}$ and CRT designed the study. OD, SI and CR coordinated the preparation of the manuscript. OD and SK carried out the preparation of the essential oils. CR carried out GC/MS analysis. NI and OD performed the in vitro tests. CV carried out the statistical analyses. All authors contributed to data analysis, read and approved the final manuscript.

\section{Acknowledgments}

The study was possible due to grant POSDRU/6/1.5/S/21 Project, ID 6540, "Pilot Programme for the Support of Scholarship PhD Students Research".

\section{Author details}

'Banat's University of Agricultural Sciences and Veterinary Medicine from Timisoara, Faculty of Veterinary Medicine, Calea Aradului no 119, Timisoara 300645, Romania. ${ }^{2}$ Department for Biomedical Sciences, Institute of Pharmacology and Toxicology, University of Veterinary Medicine Vienna, Vienna A-1210, Austria. ${ }^{3}$ Institute for Applied Botany and Pharmacognosy, University of Veterinary Medicine Vienna, Vienna A - 1210, Austria. ${ }^{4}$ Romvac, Voluntari, Bucuresti 061182, Romania.

Received: 13 September 2013 Accepted: 27 January 2014 Published: 29 January 2014

\section{References}

1. Hayat MQ, Ashraf M, Khan MA, Jabeen S: Ethnobotany of the genus Artemisia L. (Asteraceae) in Pakistan. Ethnobot Res Appl 2009, 7:147-162.

2. Hayat MQ, Ashraf M, Khan MA, Yasmin G, Shaheen N, Jabeen S: Palynological study of the Genus Artemisia (Asteraceae) and its systematic implications. Pak J Bot 2010, 42(2):751-763.

3. Pareto G: Artemisie. Ricerca ed applicazione. Q. Agric. Supp/ 1985, 2:1-261.

4. Tan RX, Zheng WF, Tang HQ: Biologically active substances from the genus Artemisia. Planta Med 1998, 64:295-302

5. Blagojevic P, Radulovic N, Palic R, Stojanovic G: Chemical composition of the essential oils of serbian wild-growing Artemisia absinthium and Artemisia vulgaris. J Agric Food Chem 2006, 54:4780-4789.

6. Juteau F, Jerkovic I, Masotti V, Milos M, Mastelic JM, Bessiere JM, Viano J: Composition and antimicrobial activity of the essential oil of Artemisia absinthium from Croatia and France. Planta Med 2003, 69:158-161.

7. Bayat M, Kousha A, Azizi Saraji A, Seyed Reza Rohani R, Nissiani M: Study effects of some kinds of standard essences over two microorganisms (Candida albicans and Gardnerella vaginalis) related to leucorrhoea disease as in vitro. World Appl. Sci. J 2008, 5(4):418-421.

8. Correa-Royero J, Tangarife V, Durán C, Stashenko E, Mesa-Arango A: In vitro antifungal activity and cytotoxic effect of essential oils and extracts of medicinal and aromatic plants against Candida krusei and Aspergillus fumigatus. Rev. Bras. Farmacogn 2010, 20(5):734-741.

9. Erdogrul OT: Antibacterial activities of some plant extracts used in folk medicine. Pharm Biol 2002, 40:269-273.

10. Fernández-Calienes Valdés A, Mendiola Martínez J, Scull Lizama R, Vermeersch $M, \operatorname{Cos} P$, Maes $L$ : In vitro anti-microbial activity of the Cuban medicinal plants Simarouba glauca $D C$, Melaleuca leucadendron $L$ and Artemisia absinthium L. Mem. Inst. Oswaldo 2008, 103(6):615-618.

11. CLSI (Clinical and Laboratory Standards Institute): Method for antifungal disk diffusion susceptibility testing of yeasts; approved guideline. In CLSI document M44-A2. Secondth edition. Pennsylavnia: Clinical and Laboratory Standards Institute, 940 West Valley Road, Suite 1400, Wayne; 2009:19087-1819.

12. Pino JA, Marbot R, Marti MP: Leaf oil of Artemisia abrotanum L. Grown in Cuba. J Essential Oil Res 2011, 23:119-120.

13. Radulovic N, Blagojevic PD, Zlatkovic BK, Palic RM: A GC/MS profile of the volatile constituents of the aerial parts of Artemisia abrotanum L. (Asteraceae) from Serbia. S Afr J Chem 2009, 62:30-32.

14. Mucciarelli M, Caramiello R, Maffei M, Chialva F: Essential oils from some Artemisia species growing spontaneously in North-west Italy. Flavour Fragrance J 1995, 10:25-32.
15. Judzentiene A, Tomi F, Casanova J: Analysis of essential oils of Artemisia absinthium L. from Lithuania by CC, GC (RI), GC-MS and ${ }^{13} \mathrm{C}$ NMR. Nat Prod Commun 2009, 4:1113-1118.

16. Vostrowsky O, Michaelis K, lhm H, Knobloch K: Das ätherische Öl von Artemisia abrotanum L. Z. Lebensmitt. Unters. Forsch 1984, 179:125-128.

17. Kowalski R, Wawrzykowski J, Zawiślak G: Analysis of essential oils and extracts from Artemisia abrotanum L. and Artemisia dracunculus L. Herba Polonica 2007, 53(3):246-254.

18. Kämäräinen-Karppinen $T$, Mäkinen $A$, Kolehmainen $S$, Hämäläinen $A$, Laine $K$, Hohtola A, Mattila S, Pirttilä AM: Overwintering, chemical variation, and genetic diversity in three vegetatively propagated lines of French tarragon (Artemisia dracunculus var. sativa). The J Horticultural Sci Biotechnol 2008, 83(6):765-769.

19. Khodakov GV, Kotikov IV, Pankovetskii VN: Component composition of essential oil from Artemisia abrotanum and A. dracunculus. Chem Natural compounds 2009, 45:905-907.

20. Lopes-Lutz D, Alviano DS, Alviano CS, Kolodziejczyk PP: Screening of chemical composition, antimicrobial and antioxidant activities of Artemisia essential oils. Phytochemistry 2008, 69(8):1732-1738,

21. Orav A, Raal A, Arak E, Müürisepp M, Kailas T: Composition of the essential oil of Artemisia absinthium L. of different geographical origin. Proc. Estonian Acad. Sci. Chem 2006, 55(3):155-165.

22. Chialva F, Liddle PAP, Doglia G: Chemotaxonomy of wormwood (Artemisia absinthium L.) I. Composition of the essential oil of several chemotypes. Z Lebensm Unters Forsch 1983, 176:363-366.

23. Kordali S, Kotan R, Mavi A, Cakir A: Determination of the chemical composition and antioxidant activity of the essential oil of Artemisia dracunculus $L$. and of the antifungal and antibacterial activities of turkish A. dracunculus, A. absinthium and Santonicum essential oil. J Agric Food Chem 2005, 53:9452-9458.

24. Jerkovic I, Mastelic M, Milos M, Juteau F, Masotti V, Viano J: Chemical variability of Artemisia vulgaris $L$. essential oils originated from the Mediterranean area of France and Croatia. Flavour Fragrance J 2003, 18:436-440.

25. Judzentiene A, Buzelyte J: Chemical composition of essential oils of Artemisia vulgaris L. (mugwort) from North Lithuania. Chemija 2006, 17(1):12-15.

26. Thao NTP, Thuy N, Hoi TM, Thai TH: Artemisia vulgaris L. from Vietnam: chemical variability and composition of the oil along the vegetative life of the plant. J. Essential Oil Res 2004, 16:358-361.

27. Brodin $\mathrm{K}$, Alahyar $\mathrm{H}$, Hedner $\mathrm{T}$, Sterner $\mathrm{O}$, Faergemann J: In vitro activity of Artemisia abrotanum extracts against Malassezia Spp., Candida albicans and Staphylococcus aureus. Acta Derm Venereol 2007, 87(6):540-542.

28. Bail S, Buchbauer G, Schmidt E, Wanner J, Slavchev A, Stoyanova A, Denkova Z, Geissler M, Jirovetz L: GC-MS-analysis, antimicrobial activities and olfactory evaluation of essential davana (Artemisia pallens Wall. ex DC) oil from India. Nat Prod Commun 2008, 3(7):1057-1062.

29. Schmidt E, Bail S, Friedl SM, Jirovetz L, Buchbauer G, Wanner J, Denkova Z, Slavchev A, Stoyanova A, Geissler M: Antimicrobial activities of single aroma compounds. Nat Prod Commun 2010, 5:1365-1368.

30. Martini MG, Bizzo HR, Moreira DDL, Neufeld PM, Miranda SN, Alviano CS, Alviano DS, Leitão SG: Chemical composition and antimicrobial activities of the essential oils from Ocimum selloi and Hesperozygis myrtoides. Nat Product Commun 2011, 6:1027-1030.

31. Pauli A, Kubeczka K-H: Antimicrobial properties of volatile phenylpropanes. Nat Prod Commun 2010, 5:1387-1394.

32. Popović V, Petrović S, Pavlović M, Milenković M, Couladis M, Tzakou O, Duraki Š, Niketić M: Essential oil from the underground parts of Laserpitium zernyi: potential source of a-bisabolol and its antimicrobial activity. Nat. Pro. Commun 2010, 5:307-310.

33. Verdian-Rizi MR, Sadat-Ebrahimi E, Hadjiakhoondi A, Fazeli MR, Pirali Hamedani M: Chemical composition and antimicrobial activity of Artemisia annua L. essential oil from Iran. J. Med. Plants 2008, 7(4):58-62.

34. Damjanovic-Vratnica B, Dakov T, Šukovic D, Damjanovic J: Chemical composition and antimicrobial activity of essential oil of wild-growing Salvia officinalis L. from Montenegro. J Essential Oil-Bearing Plants 2008, 11(1):79-89.

35. Celikel N, Kavas G: Antimicrobial properties of some essential oils against some pathogenic microorganisms. Czech J Food Sci 2008, 26:174-181.

36. Damjanovic-Vratnica B, Dakov T, Šukovic D, Damjanovic J: Antimicrobial effect of essential oil isolated from Eucalyptus globulus Labill. from Montenegro. Czech J Food Sci 2011, 29:277-284. 
37. Ezzat SM: In vitro inhibition of Candida albicans growth by plant extracts and essential oils. World J Microbiol Biotechnol 2001, 17:757-759.

38. Kuete V, Metuno R, Ngameni B, Tsafack AM, Ngandeu F, Fotso GW, Bezabih M, Etoa F, Ngadjui BT, Abegaz BM, Beng VP: Antimicrobial activity of the methanolic extracts and compounds from Treculia obovoidea (Moraceae). J Ethnopharmacol 2007, 112:531-536.

39. European Directorate for the Quality of Medicines (EDQM): The European Pharmacopoeia. 6th edition. Strasbourg: BDC Ed; 2008 (2.8.12.) (ISBN: 9789287160546)

40. Adams RP: Identification of essential oil components by gas chromatography/ quadrupole mass spectroscopy. 4th edition. Carol Stream, IL: Allured; 2007.

41. McLafferty FW: Registry of mass spectral data. 5th edition. New York: John Wiley \& Sons; 1989.

42. Iten F, Saller R, Abel G, Reichling J: Additive effects of the active components of the essential oil of Thymus vulgaris - chemotype carvacrol. Planta Med 2009, 75:1231-1236.

doi:10.1186/1752-153X-8-6

Cite this article as: Obistioiu et al:: Chemical characterization by GC-MS and in vitro activity against Candida albicans of volatile

fractions prepared from Artemisia dracunculus, Artemisia abrotanum, Artemisia absinthium and Artemisia vulgaris. Chemistry Central Journal 2014 8:6.

\section{Publish with ChemistryCentral and every scientist can read your work free of charge \\ "Open access provides opportunities to our colleagues in other parts of the globe, by allowing anyone to view the content free of charge." \\ W. Jeffery Hurst, The Hershey Company. \\ - available free of charge to the entire scientific community \\ - peer reviewed and published immediately upon acceptance \\ - cited in PubMed and archived on PubMed Central \\ - yours - you keep the copyright \\ Submit your manuscript here: \\ http://www.chemistrycentral.com/manuscript/<smiles>c1ccccc1</smiles> \\ Chemistry Central}

